\title{
Anatomo-morphological stem features of spring bread wheat varieties
}

\author{
Ageeva E.V. ${ }^{*}{ }^{*}$, Leonova I.N. ${ }^{2}$, Salina E.A. ${ }^{2}$, Likhenko I.E. $^{1}$ \\ ${ }^{1}$ Siberian Research Institute of Plant Production and Breeding - Branch of the Institute of Cytology \\ and Genetics, SB RAS, Novosibirsk, Russia \\ ${ }^{2}$ Institute of Cytology and Genetics, SB RAS, Novosibirsk, Russia \\ *e-mail:elenakolomeec@mail.ru
}

Using the anatomical method, the study of the main parameters of the internal stem structure of Siberian bread wheat varieties (internode diameter, mechanical layer thickness, number and diameter of vascular bundles of parenchyma) was performed in order to assess their impact on the resistance to lodging. The assessment focused on the study of the first two internodes located under the ear: EN1 and EN2, since these internodes reflect the genotypic features of the plant anatomy. An increase in the number of parenchyma vascular bundles from top to bottom was established. The number of bundles in EN1 varied from 14 to 24, while in EN2 from 16 to 33. On average, the number of bundles from EN1 to EN2 increased by 8.0 for the studied varieties. The decrease in the diameter of the vascular bundles is compensated by an increase in their number. This pattern was clearly observed in cultivars Novosibirskaya 18 (224 $\mu \mathrm{m}$ and 18 bundles), Obskaya 2 (215 and 19), Trizo (228 and 18) and Bel (228 and 24) in the EN1; in Novosibirskaya 29 (268 and 26), Chernyava 13 (270 and 28), Novosibirskaya 18 (262 and 28) and Velut (239 and 29) in EN2. Bel and Novosibirsk 31 were found to differ from the other cultivars on the thickness of the mechanical layer. According to the combination of the stem internal structures (the number and diameter of vascular bundles and the stem diameter) the varieties Trizo, Velut, Novosibirskaya 29 and Novosibirskaya 31 were shown to overcome the others. The Obskaya 2 and Bel differed in a large number of vascular bundles of small diameter with a large stem diameter. Significant correlations $(\mathrm{r}=0.91)$ was established between the stem length and the length of the EN1. A positive correlation ( $\mathrm{r}=0.59-0.37)$ was observed between the number of vascular bundles and resistance to lodging. The correlation coefficient between the stem diameter in EN1, EN2 and resistance to lodging does not exceed 0.21 . The obtained results can be used in the evaluation of spring bread wheat for resistance to lodging. 